\title{
Predicting object mass nouns across languages
}

\author{
Kurt Erbach*
}

\begin{abstract}
The hypothesis explored in this paper is that the amount of object mass nouns (e.g. furniture, jewelry) in a given language is related to the amount of morphosyntax that indicates the countability nouns (e.g. many, much) in that language. This hypothesis, together with the analysis of Sutton \& Filip (2016) best captures the occurrence of object mass nouns across languages. The analysis of Sutton \& Filip (2016) accurately predicts which class of nouns will have object mass nouns across languages_collective artifacts—and the novel hypothesis provides a means of predicting the amount of object mass nouns in a given language: languages with many morphosyntactic reflexes of the mass/count distinction will likewise have many object mass nouns-e.g. English—and languages with few morphosyntactic reflexes of the mass/count distinction will likewise have few object mass nouns-e.g. Greek, Hungarian, and Japanese.
\end{abstract}

Keywords. mass; count; countability; English; Greek; Hungarian; Japanese

1. Introduction. The main idea argued for in this paper is that, across languages, the amount of object mass nouns in a language is related to the amount of morphosyntax that indicates the countability of nouns in that language. This idea is in direct conflict with certain theories of countability that make alternative crosslingluistic predictions. For example Chierchia (2015) argues that certain languages-e.g. those like Greek and Japanese-cannot have object mass nouns, despite having morphosyntactic environments that indicate the countability of nouns. Alternatively, Rothstein (2010) predicts that languages can have very large numbers of object mass nouns so long as these nouns can be counted with classifiers-e.g. those like Hungarian and Japanese. The grounds for motivating a hypothesis alternative to those argued for by, for example Chierchia (2015) and Rothstein (2010), include taking into account recent research that suggests that each of the aforementioned languages have both a relatively small amount of object mass nouns and a relatively small amount of morphosyntactic environments that indicate the countability of nouns. As suggested by Chierchia (2010), object mass nouns constitute a testing ground for theories of the mass/count distinction. The presence of object mass nouns in Greek, Hungarian, and Japanese constitutes evidence that militates against certain analyses of the mass/count distinction, namely those of Chierchia (2015) and Rothstein (2010), and this evidence is compatible with analyses like that of Sutton \& Filip (2016). More generally, this evidence supports theories that assume that object mass nouns arise among a relatively small notional class of nouns - collective artifacts — which can be analyzed as denoting overlapping individuated entities as opposed to assuming that object mass nouns can arise among all nouns that denote objects.

2. Background. Object mass nouns get their name from their two primary properties: (i) they are mass nouns that (ii) refer to objects. In English, mass nouns have long been identified as

\footnotetext{
*Kurt Erbach, University of Bonn (kerbach@uni-bonn.de). Special thanks to Hana Filip, Scott Grimm, and Peter Sutton for advising my thesis, which the present paper draws from, thanks also to Yasu Sudo for further comments on this work. This research was funded by DFG Collaborative Research Centre 991: The Structure of Representationsin Language, Cognition, and Science, Project C09: A frame-based analysis of countability.
} 
those that occur with quantifiers like much and little, but do not straightforwardly ${ }^{1}$ occur with numerical modifiers, plural morphology, determiners like $a(n)$, these, and those, or quantifiers like each, every, several, few, many, etc. Nouns like furniture, equipment, soil, and mud each qualify as mass nouns under these criteria.

(1) *three muds, *three furniture

(Rothstein 2010; p. 346)

(2) *every/several furniture(s)

(Rothstein 2010; p. 347)

Object mass nouns like furniture, equipment, luggage, and jewelry have been identified by this name, or others like 'fake mass nouns' (Landman 2011) and 'naturally atomic mass nouns' (Rothstein 2010), at least since Barner \& Snedeker (2005) showed that these nouns are susceptible to cardinality based quantity comparison, while nouns that refer to substances are not-e.g. soil, mud, mustard, and clay. Additionally, Rothstein (2010) and Schwarzschild (2011) both observed that the same object denoting nouns are felicitous with predicates like big, round, long, and large, which Schwarzschild (1996) named 'stubbornly distributive' because of the fact that they require multiple individuals to distribute over. This stubborn distributivity is illustrated by the fact that big felicitously modifies furniture, which refers to multiple individuals, in (3), while this and other predicates do not felicitously modify nouns like wine, snow, cocaine, and traffic, which according to Schwarzschild (2011) do not refer to multiple individuals (4).

(3) a. The furniture in our house is big.

b. In a department store: 'The big furniture is on the third floor.'

c. To movers who are emptying the house: 'Please take the big furniture down first.'

d. 'Don't buy big furniture, the stairs are too narrow to carry it up.'

(Rothstein 2010; p. 360)

(4) a. ?The wine is big. ${ }^{2}$

b. ?The snow is round.

c. ?The cocaine was long.

d. ?The traffic is large.

(Schwarzschild 2011; p. 5)

Before these semantic tests were observed by Barner \& Snedeker (2005); Rothstein (2010) and Schwarzschild (2011), Chierchia (1998) and Gillon (1999) observed that mass nouns in this class often have count counterparts: luggage, suitcases; footwear, shoes; etc. Chierchia (1998) also noted that these nouns notionally refer to objects. For Chierchia (1998) and many others, object is a technical term for "bodies that are cohesive, bounded, spatiotemporally continuous, and solid or substantial; they move as connected wholes, independently of one another, on connected paths though unoccupied space" (Soja et al. 1991; p. 183). 'Collective artifacts' is a term used by Sutton \& Filip (2016, 2018); Erbach et al. (2019) among others to refer to all nouns, mass and count, in this notional class, though the term 'collective aggregates' has also been used (e.g. Grimm 2012).

Given the identification of object mass nouns requires (i) a grammatical test for being a mass noun, and (ii) a semantic test for distinguishing object mass nouns from other mass

\footnotetext{
${ }^{1}$ By straightforwardly I mean they do not combine without occurring in a measure, portion, or kind phrase, or without being coerced into such an interpretation if it is possible to do so.

${ }^{2}$ This judgment pertains to a judgment in size, as opposed to one where the flavor of the wine is being described as, e.g. 'full bodied' or otherwise.
} 
nouns, one might expect to be able to find object mass nouns in any language in which such tests are feasible. While certain semantic theories of countability allow for the possibility of finding object mass nouns in any language (e.g. Rothstein 2010; Sutton \& Filip 2016), others explicitly predict that certain languages should have no object mass nouns at all because of other properties of their respective nominal systems (e.g. Chierchia 2010, 2015; Rothstein 2017). The following subsections will review three semantic theories of countability, each of which makes different predictions about the extent to which one might find object mass nouns across languages. There have been many other treatments of countability and object mass nouns in formal semantics Landman (2011, 2016); Grimm (2012); Grimm \& Levin (Submitted), but I will focus on three that have made particular crosslinguistic predictions and which have been applied to typologically distinct languages.

2.1. SEMANTIC COPY CAT. Object mass nouns, under the analysis of Chierchia (2015) denote stable atoms, but they also copy substance denoting nouns in denoting a singleton property. Stable atoms are those that are atomic, in every accessible world, as defined in (5), and unstable atoms are those that are not atomic in every accessible world, (6); when not atomic, what is referred to is a plurality.

$$
\begin{gathered}
\operatorname{AT}(\mathrm{P})=\lambda \mathrm{w} \lambda \mathrm{x}\left[\mathrm{P}_{\mathrm{w}}(\mathrm{x}) \wedge \forall \mathrm{w}^{\prime} \in \mathrm{K}_{\mathrm{w}} \forall \mathrm{z}\left[\mathrm{P}_{\mathrm{w}^{\prime}}(\mathrm{z}) \wedge \mathrm{z} \leq \mathrm{x} \rightarrow \mathrm{z}=\mathrm{x}\right]\right] \\
\operatorname{MASS}(\mathrm{P})=\forall w \forall x\left[P_{w}(x) \wedge \forall z\left[P_{w}(z) \wedge z \leq x \rightarrow z=x\right] \rightarrow\right. \\
\square \forall y[P(y) \wedge y \leq x \rightarrow y=x]]
\end{gathered}
$$

(Chierchia 2015; p. 161)

(Chierchia 2015; p. 160)

Leaning on the definition of objects from Soja et al. (1991), nouns that refer to objects denote stable atoms, and those that do not refer to unstable atoms. For Chierchia (2015), the difference between stably atomic and not stably atomic nouns is not enough for characterizing the difference between count nouns and mass nouns respectively, because this difference alone cannot explain how mass nouns can be grammatically singular: since mass nouns refer to unstable atoms, in some worlds they refer to pluralities and so their reference does not match the definition of singular number marking that Chierchia (2015) assumes to hold in English, namely that singular nouns must be atomic (7).

$$
\begin{array}{ll}
\text { a. } & \mathrm{PL}(\mathrm{P})=\mathrm{P} \text { if }{ }^{*} \mathrm{AT}(\mathrm{P})=\mathrm{P} \\
\text { b. } & \mathrm{SG}(\mathrm{P})=\mathrm{P} \text { if } \mathrm{AT}(\mathrm{P})=\mathrm{P}
\end{array}
$$

(Chierchia 2015; p. 164)

To account for the singular morphology of mass nouns, Chierchia (2015) assumes that mass nouns denote a singleton property, that is, the sum of all unstable atoms. With the definitions of atomicity and number marking taken together, Chierchia (2015) characterizes singular count nouns as those that each refer to a set of stable atoms, while mass nouns have singular morphology and refer to a stably atomic singleton property. Object mass nouns have the requisite properties to be count nouns, but copy the behavior of mass nouns in denoting a singleton property as a matter of lexical choice.

From Chierchia's (2015) theory of the mass/count distinction, it follows that any language with number marking defined as above can potentially have object mass nouns, while those without such number marking cannot. Examples of languages that do not have this sort of number marking are Greek and Japanese. In Greek, mass nouns can take plural morphology, as in (8), to express something like 'much' or 'spread'/'scattered' (Tsoulas 2008; Alexiadou 2011; Kouneli 2019), so it cannot be the case that (7) also defines number marking in Greek. 
Trehoun nera apo to tavani

Drip.3RD.PL waterPL.NEUT.NOM from the ceilingNEUT.SG

'Water is dripping from the ceiling'

(Tsoulas 2008; p. 133)

Japanese, on the other hand, does not have obligatory plural morphology, and classifiers are used in counting constructions, (9), since all nouns are assumed to refer to kinds, so Japanese likewise cannot have number marking defined as in (7).

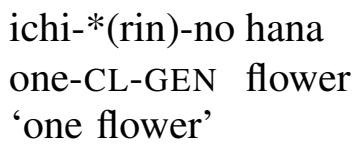

Because neither Greek nor Japanese have number marking like that in English, which gives rise to object mass nouns, in Chierchia's (2015) analysis of countability, it is predicted that these languages should have no object mass nouns. Chierchia (2015) provides analyses nominal systems that give rise to the properties had by languages like Greek and Japanese, but in these analyses, there are no other mechanisms like the copy cat effect assumed to exist in English, that could give rise to object mass nouns. Evidence of object mass nouns in either of these languages would therefore militate against Chierchia's (2015) analysis.

2.2. No COUNTING CONTEXT. In Rothstein's (2010) analysis of countability, object mass nouns denote natural atoms and sums thereof, but they are not indexed to a counting context and are therefore mass. Rather than adopting the definition of objects given by Soja et al.(1991), Rothstein (2010) uses the term 'natural atoms' and defines it as in (10), which states that natural atoms are atoms in all contexts.

(10) Natural atomicity:

If $\mathrm{N}$ is a naturally atomic predicate then:

$\forall \mathrm{x} \forall \mathrm{k} \forall \mathrm{k}^{\prime}\left[\mathrm{x} \in \pi_{1}\left(\mathrm{~N}_{\mathrm{k}}\right) \wedge \mathrm{x} \in \pi_{1}\left(* \mathrm{~N}_{\mathrm{k}}, \rightarrow \pi_{1}\left(\mathrm{~N}_{\mathrm{k}},\right)\right)\right]$

'If $\mathrm{N}$ is naturally atomic, then for any two contexts $\mathrm{k}$ and $\mathrm{k}$ ', if $\mathrm{x}$ is an atom of $\mathrm{N}_{\mathrm{k}}$, and $\mathrm{x}$ is in the denotation of $\mathrm{N}_{\mathrm{k}}$, $\mathrm{x}$ is also an atom in $\mathrm{N}_{\mathrm{k}}$,

(Rothstein 2010; p. 373)

Noun roots are assumed to express general number number-i.e. they refer to entities and sums thereof-and mass nouns are equivalent in denotation to roots.

Rothstein (2010) assumes that count nouns are typally distinct from mass nouns, and what makes them countable is the fact that they are indexed to counting contexts, which denote the entities that can be counted. Rothstein (2010) does note delineate any limitation on which nouns can be mass or count in her analysis, and she uses this to make predictions about the behavior of nouns in languages like Japanese. Rothstein (2010) assumes that nouns in languages like Japanese are all mass, and that the classifier in counting constructions like (9) indexes the mass noun to a counting context and therefore the classifier + noun construction can combine with a numerical like ichi ('one'). Within this analysis, all nouns in these languages that refer to natural atoms are essentially object mass nouns.

Schvarcz \& Rothstein (2017) build on Rothstein (2010) to propose an analysis of Hungarian in which it, like Japanese, has a very large number of object mass nouns. Hungarian allows nouns that refer to objects (or, natural atoms) to be counted in two ways: either directly as in English (though without plural morphology), or with a classifier as in Japanese (again without plural morphology). 
Schvarcz \& Rothstein (2017) assume that object denoting nouns that can be counted in both ways have two distinct denotations: one that is like a singular count noun in English, which refers to entities indexed to a counting context, and one that is like an object mass noun in English (or an object denoting noun in Japanese), which refers to natural atoms and sums thereof but is not indexed to a counting context.

While Rothstein (2010) and Schvarcz \& Rothstein (2017) assume there are very large numbers of object mass nouns in languages like Hungarian and Japanese, such patterns are unlike those made for other languages in which seem to have a restricted set of object mass nouns. Evidence of a restricted set of nouns having distinct object mass characteristics outside of counting constructions, in languages like Japanese and Hungarian, would militate against Rothstein's (2010) assumption that potentially any object denoting noun can be object mass.

2.3. INDIVIDUATION WITHOUT SPECIFICATION. Under the analsysis of Sutton \& Filip (2016), object mass nouns are those that denote individuated entities which overlap in the null counting context in which these nouns are interpreted. Sutton and Filip's (2016) individuation function, IND, identifies the entities in the denotation of a noun that could potentially be counted as 'one' (this idea being inspired by Rothstein's (2010) notion of counting context), though, crucially, these individuated entities might overlap. For example, the individuation function applied to the root KITCHENWARE, identifies all of the items of kitchenware that could potentially be counted as 'one', and this set of individuated entities will denote items like tea cups, saucers, and entire tea sets, each of which could count as one, and which overlap given the tea cups and saucers are part of the tea set. The various ways of counting items of kitchenware in different contexts is inspired by the notion of variants from Landman (2011) (see also Krifka 2009). Within this analysis, only nouns whose set of individuated entities overlap could be object mass nouns. IND(DOG), for example, could not be an object mass noun, because no sum of dogs could be counted as 'one'. At the same time, not all overlapping sets of individuated entities give rise to object mass nouns, because Sutton \& Filip (2016) assume a second operation in the denotation of nouns, $\mathrm{c}_{\mathrm{i}}$, which specifies which of the individuated entities are to be counted in the context of use. If a denotation of the noun like kitchenware is saturated with the null counting context, $\mathrm{c}_{0}$, which is the identity function on the set of individuated entities and does not specify a set of entities that are disjoint from one another, then it will be an object mass noun, because the counting operation is assumed to require nouns to have a non-disjoint denotation, and, as we know, the set of individuated entities in the denotation of kitchenware is overlapping. If the denotation contains a specific counting context, which specifies a disjoint denotation, then the noun is

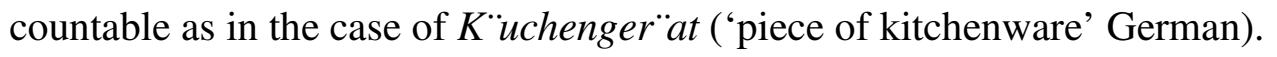

Sutton and Filip's (2016) analysis predicts that the only nouns that have the potential to be object mass nouns are collective artifact nouns-i.e. those denote an overlapping set of individuated entities when the IND function is applied to the root. This also means that, crosslinguistically, only a restricted set of nouns have the potential to be object mass nouns. In languages with nominal systems like those in Greek, Hungarian, and Japanese, the potential number of object is not any larger than that in English. This analysis is compatible with that 
of Chierchia (2015), because it allows for there to be no mass nouns in a given language. One difference between the two is that Chierchia (2015) does not give clear restrictions on which object denoting nouns might become object mass, while Sutton \& Filip (2016) do. Conversely, Sutton \& Filip (2016) do not adopt a strong definition of number marking like that of Chierchia (2015), so the former analysis is compatible with the existence of object mass nouns in languages like Greek, Hungarian, and Japanese, while the latter predicts that object mass nouns should not occur in such languages. At the same time, Sutton and Filip's (2016) analysis is not compatible with that of Rothstein (2010), given the latter assumes that all object denoting nouns can be object mass, and essentially assumes this is the case in Hungarian and Japanese. This latter assumption is not uncontroversial, and Rothstein (2017) essentially claims the opposite for languages like Japanese, namely that no noun that refers to objects is object mass, rather she assumes that all nouns in such languages denote kinds.

To summarize Section 2, object mass nouns are defined in a particular way that does not entail any restrictions on the amount of object mass nouns in one particular kind of language or another. So long as a language has a means of distinguishing mass nouns and count nouns, and a means of determining that certain mass nouns have object reference, it is possible to test for object mass nouns in that language. From analyses of the mass/count distinction, it often follows that one can assume how many object mass nouns a language may or may not have. Chierchia (2015) predicts that Greek and languages like Japanese should have no object mass nouns, Rothstein (2010) assumes that all object denoting nouns in languages like Japanese are object mass and a similar analysis is made by Schvarcz \& Rothstein (2017) with respect to Hungarian, and Sutton \& Filip (2016) predict that the number of object mass nouns in a language can be no higher than the number of nouns that refer to collective artifacts in that language. Each of these analyses paint very different pictures of the possible numbers of object mass nouns in a given language, and in-depth analysis of object mass nouns in typologically distinct languages is necessary to put these theories to the test.

3. Proposal. The novel proposal argued for in this paper is that the amount of object mass nouns in a language corresponds to the amount of morphosyntax that reveals the countability of the noun. The amount of object mass nouns in a language might be tied to the amount of morphosyntax that rreveasls the countability of each noun, because the morphosyntax in which a noun appears has been shown to be indicators of the countability of a noun for children as young as age 3 Gordon (1985). In other words, if learners do not constantly experience nouns being used in particular morphosyntax - i.e that which enforces either count encoding or mass encoding - then when it comes to counting, they may decide whether or not a nouns is countable on perceptual features, like whether or not a noun refers to objects or a substance. On the other hand, if a learners constantly experience nouns being used in morphosyntax that enforces either count or mass encoding, then speakers are less likely rely on perceptual features to determine countably and that language will be more likely to have a larger number object mass nouns.

This proposal aims to be a middle of the road approach, compared to that argued for by Chierchia (2015) and Rothstein (2010), which respectively argue that certain languages should have no object mass nouns at all, or that they should have as many nouns be object mass as is possible. Recall that the way in which object mass nouns are identified relies on (i) a noun's behavior in a large number of morphosyntactic environments that indicate whether a noun 
is count or mass, and (ii) whether or not that noun can be determined to refer to objects via semantic tests like quantity comparison or felicity with stubbornly distributive predicates. Because these characteristics are not, per se, strictly tied to grammatical features like number marking or counting constructions alone, an analysis in which the underlying characteristics of object mass nouns are not rooted these particular features is possibly more likely to be able to capture the existence of object mass nouns in languages with nominal systems that vary from English with respect to the manifestation of the mass/count distinction. Because the analysis of Sutton \& Filip (2016) is tied to the way entities in the denotations of nouns are counted rather than number marking or counting constructions, it is the most compatible with a middle of the road hypothesis about the possible amount of object mass nouns in a given language, namely one where this amount is related to the amount of morphosyntax that reveals the countability of nouns.

The analysis of Sutton \& Filip (2016), together with the novel proposal of this paper predicts very different patterns compared to the respective analyses of Chierchia (2015) and Rothstein (2010). Rather than seeing the largest number of nouns with object mass denotations in languages where nouns that refer to objects can be counted with classifiers, as assumed by Rothstein (2010), and rather than seeing no object mass nouns in languages that do not have a strong definition of number marking as in (7), what we should expect to see across languages is that the largest possible amount of object mass nouns is equivalent to the amount of collective artifact nouns, that is, those whose set of individuated entities is overlapping. We should also expect to see that a language is only likely to approach this amount if there is also a large amount of morphosyntax that overtly indicates the countability of a noun. In languages where there are few morphosyntactic environments that indicate the countability of a noun, it will be more likely that there are few object mass nouns, if there are any at all.

4. Data. Each of the aforementioned theories of countability are supported by the data reviewed above, though more recent investigations have brought additional data into discussion. In this section, data from English is reviewed first as a way of delineating known characteristics of the mass/count distinction and the number of object mass nouns in English. Subsequently, data from Greek, Hungarian, and Japanese will be reviewed and discussed with respect to the consequences it presents for these particular theories

4.1. ENGLISH. The characteristics of the mass/count distinction in English have been documented and discussed widely, though Chierchia (1998) provides one of the most comprehensive lists. He provides 13 different ways that one might 'test' for the countability of a given noun: numerical determiners, number marking, obligatory classifiers for counting, a(n), both, each, every, few, a few, many, several, little, and much. As exemplified in (12), some English nouns straightforwardly take plural morphology - e.g. shoe-with others do not-e.g. footwear, blood.

(12) a. There are shoes in this store.

b. *There are footwears in this store.

c. *There are bloods on the wall.

(Chierchia 1998; p. 55)

While the list from Chierchia (1998) is one of the largest, it certainly does not contain all 'tests' for countability, as it does not contain demonstrative determiners like these and those, counting phrases like dozens of or hundreds of, etc.

In addition to having an incomplete list of known tests for countability, the list of known 
object mass nouns is also incomplete. Citing those tested respectively by Barner \& Snedeker (2005), Rothstein (2010), and Schwarzschild (2011), the number would be only seven, including furniture, clothing, jewelry, silverware, mail, luggage, and equipment. Erbach (2020) gives a list of more than 40 additional nouns, including inventory, teaware, swag, etc., that may prove to be object mass when tested, therefore bringing the number of possible object mass nouns in English up to around 50 .

4.2. GREEK. In his analysis of plural mass nouns in Greek, Tsoulas (2008) notes only two tests for countability: numerical determiners and the determiner kathe ('every') are only straightforwardly felicitous with count nouns. Mass nouns, on the other hand, are not straightforwardly used with either.

* Kathe nero ine ahromo every water is colorless 'Every water is colorless.'

(Tsoulas 2008; p. 137)

In addition to there having been only two tests for countability discussed with respect to Greek, there have only been two Greek nouns labeled object mass. Alexiadou (2015) uses unacceptability with plural morphology as a test for mass nouns and felicity with stubbornly distributive predicates as a test for objecthood, showing that epiplosi ('furniture') and ruhismos ('clothing') are object mass nouns in Greek

(14) I epiplosi sto domatio ine strogili. the furniture in room is round 'The furniture in the room is round.'

(Alexiadou 2015; p. 15)

$$
\begin{aligned}
& \text { *ruhismi } \\
& \text { *clothings }
\end{aligned}
$$

(Alexiadou 2015; p. 15)

The Greek data, as the English data is likely incomplete, though it seems the number of object mass nouns in Greek may indeed be particularly low, as Tsoulas (2008) notes that they are particularly hard to identify on account of the fact that nouns known to be object mass in English are countable in Greek.

4.3. Hungarian. With respect to Hungarian, Schvarcz \& Rothstein (2017) describe three tests for countability in which count nouns are felicitous but mass nouns are not: plural morphology, direct counting, and the count WH-determiner hány ('how many'), and Erbach (2020) shows that egy par ('a couple of') can be characterized as a test for countability as well.

$$
\text { három (darab) könyv }
$$

three $\mathrm{CL}_{\text {general }}$ book

'three books'

(Schvarcz \& Rothstein 2017; p. 185)

$$
\begin{aligned}
& \text { *három *(darab) sár } \\
& \text { three } \mathrm{CL}_{\text {general }} \text { mud } \\
& \text { 'three pieces of mud' }
\end{aligned}
$$

(Schvarcz \& Rothstein 2017; p. 194)

Object mass nouns in Hungarian can be identified by infelicity with respect to the tests above which all select for count nouns. Erbach et al. (2019) shows that lószer ('ammunition'), felszerelés ('equipment'), and csomagolás ('packaging') are genuine mass nouns, patterning with kosz ('dirt') in examples like (17) rather than with nouns like könyv ('book') as in (16). The current 
state of research on countability thus shows that Hungarian has four tests for countability and three object mass nouns.

Crucially, nouns like könyv ('book), which Schvarcz \& Rothstein (2017), call dual life on account of their assumption that these have an object mass denotation in addition to a count denotation, do not behave like other mass nouns with respect to all countability tests. For example, one commonly discussed characteristic of mass predicates is that they have cumulative reference, namely that they can refer to the sum of any two entities that they can refer to separately (Link 1983; Krifka 1989; Rothstein 2010; Sutton \& Filip 2016; among others). Hungarian object mass nouns have this property as well, though the nouns that Schvarcz \& Rothstein (2017) call dual life do not. For example, a lószer ('the ammunition') can be used to refer to individual pieces of ammunition or sums thereof, while a könyv ('the book') can only refer to individual books suggesting that it does not have an object mass denotation available in this sort of construction.

A lőszer 2kg-ot nyom. the ammunition $2 \mathrm{~kg}$-ACC weigh

'The ammunition weighs 2 kilograms.' (e.g. one or several pieces) (Erbach et al. 2019)

\section{A könyv $2 \mathrm{~kg}$-ot nyom.} the book $2 \mathrm{~kg}$-ACC weigh

'The book weighs 2 kilograms.' (only refers to one book)

(Erbach et al. 2019)

Data such as (18) and (19) call into question the characterization of nouns like könyv ('book') as dual life. Erbach et al. (2019) propose an alternative analysis to that of Schvarcz \& Rothstein (2017), arguing that classifiers restrict counting rather than shift mass nouns to countable predicates, meaning that nouns like könyv ('book') are strictly count nouns, rather than dual life, and the number of object mass nouns in Hungarian is relatively small.

4.4 JAPANESE. Given that counting occurs with classifiers in Japanese, it is generally assumed that Japanese has no grammaticized lexical mass/count distinction, rather, all nouns in such languages are of the same semantic type (Chierchia 1998; Cheng \& Sybesma 1998; Muromatsu 2003; Nemoto 2005; Li 2011; Rothstein 2017). At the same time, however, Sudo (2015) shows that Japanese has at least five morphosyntactic environments to distinguish countable nouns from non-countable nouns: numerical determiners and four count determiners. For example, the quantifier phrase nan-byakut-toiuu ('hundreds of') is felicitous with nouns like komento ('comment') but infelicitous with nouns like ase ('sweat').

(20) a. sono tookoo-ni nan-byaku-toiuu komento-ga tsuita.

that post-TO what-100-say comment-NOM provided.

'That post got hundreds of comments.'

b. Taro-wa nan-byaku-toiuu ase-o kaita

Taro-TOP what-100-say sweat-ACC secreted (intended) 'Taro sweated a lot.'

(Sudo 2015; p. 5)

Crucially, Sudo (2015) intentionally uses the terms 'countable' and 'noncountable' rather than 'count' and 'mass' respectively to distinguish this behavior of nouns in Japanese from their counterparts in other languages that can be straightforwardly tied to a mass/count distinction, rather than assuming that the phenomena are identical in nature. 
Building on the observations of Sudo (2015), Erbach et al. (2020) show that there is evidence to support the idea that Japanese has nouns that display the characteristic behavior of object mass nouns. In an acceptability judgment task and follow-up consultation, Erbach et al. (2020) found that shinamono ('wares/articles') and kattamono ('shopped goods') are judged to be the least felicitous when composed with nan-byaku-toiuu ('hundreds of') in sentences. These nouns are also judged according to cardinality in quantity comparison tasks, and therefore they display both characteristics of object mass nouns.

5. Analysis. The evidence of object mass nouns in English, Greek, Hungarian, and Japanese presented in Section 4 supports the proposal of this paper that larger amounts of object mass nouns are more likely to be found in languages with larger amounts of morphosyntactic environments in which the countability of a noun is overtly observable. Table 1 summarizes the data described above, and it shows that the language with the largest approximate number of morphosyntactic environments in which the countability of a noun is overtly observable, or 'tests', likewise has the largest number of object mass nouns. Conversely, the language with the smallest amount of tests, Greek, has the smallest amount of object mass nouns. Hungarian and Japanese pattern very closely to Greek in having both few tests for countability and few object mass nouns.

\begin{tabular}{lcc} 
Language & Tests & Object Mass Nouns \\
\hline English & 13 & 50 \\
Greek & 2 & 2 \\
Hungarian & 4 & 3 \\
Japanese & 5 & 2 \\
\hline
\end{tabular}

Table 1. Approximate amounts of countably tests and object mass nouns

The predicted pattern pertaining to amount of morphosyntactic environments in which the countability of a noun is indicated and the amount of object mass nouns in a language is directly observable in recent research on countability and object mass nouns in English, Greek, Hungarian, and Japanese.

A consequence of observing this pattern and confirming the main hypothesis of this paper is that support is provided for the analysis of object mass nouns argued for by Sutton \& Filip (2016), while the respective analyses of Chierchia (2010) and Rothstein (2017) are militated against. Recall that Sutton \& Filip (2016) expect to find object mass nouns among nouns that denote overlapping individuated entities at the null counting context. More plainly, Sutton \& Filip (2016) predict that object mass nouns will only refer to collective artifact nouns like kitchenware, equipment, etc. whose individuals can be counted in multiple ways-recall a tea set could be one item of kitchenware or many-while nouns that refer to things like cats will never be object mass across languages because individual cats can only be counted in one way: each cat is one cat. In English, Greek, Hungarian, and Japanese, the predictions of Sutton \& Filip (2016) are borne out.

Conversely, the data discussed herein militates against the respective analyses of Chierchia (2015) and Rothstein (2010). Under Chierchia's (2015) analysis, the only language among these four that should have any object mass nouns at all is English, given the number marking in English can be assumed to be defined with respect to atomicity, and one can assume that object mass nouns arise due to a copy cat effect whereby object denoting nouns that could be count choose to copy substance denoting nouns in denoting a singleton property. This definition 
of number marking cannot not be upheld in Greek, where mass nouns straightforwardly take plural morphology, Hungarian, where singular nouns are used in all counting constructions and also with quantifiers, or Japanese, where there is no obligatory number marking at all. Given Chierchia's (2015) analysis of number marking and object mass nouns in English cannot be applied to Greek, Hungarian, and Japanese, the analysis cannot account for the data above.

Recall that Rothstein (2010) would predict that there are many object mass nouns in Hungarian and Japanese since object denoting nouns in these languages can be counted with classifiers. However, as observed in the respective sections on Hungarian and Japanese data, each of these languages have a subset of object denoting nouns that display mass noun behavior in more contexts than those observed by Rothstein (2010) and Schvarcz \& Rothstein (2017), while the nouns that are assumed by Rothstein (2010) and Schvarcz \& Rothstein (2017) to have an object mass denotation do not display object mass behavior in these other contexts. In other words, it seems that neither Hungarian nor Japanese have a very large amount of object mass nouns, as would be predicted by Rothstein (2010) given few nouns display mass behavior in all relevant contexts.

6. Conclusion. In summary, looking at the relative numbers of morphosyntactic environments in which a noun's countability is overly specified and the number of object mass nouns across languages, the pattern that emerges fits with the hypothesis argued for in this paper: The number of object mass nouns in a language is related to the number of morphosyntactic environments in which a noun's countability is overtly specified. Comparing the relative amounts of these phenomena in English, Greek, Hungarian, and Japanese reveals that the language with the largest amount of morphosyntactic environments in which a noun's countability is specified, namely English, is indeed the language with the largest amount of object mass nouns, while languages with much smaller amounts of morphosyntactic environments in which a noun's countability is specified, namely Greek, Hungarian, and Japanese, likewise have much smaller amounts of object mass nouns.

This hypothesis fits with acquisition research that shows that the countability of a noun can be observed by children as young as three years old by looking at the morphosyntax in which a noun occurs (e.g. Gordon 1985). This hypothesis also fits with the analysis of object mass nouns and countability argued for by Sutton \& Filip (2016), given they do not predict that any particular type of language should have zero object mass nouns (unlike Chierchia 2015), nor do they predict that any particular language should have a very large number of object mass nouns (unlike Rothstein 2010). Instead, Sutton \& Filip (2016) predict that object mass nouns should be found among nouns that can refer to individuals that overlap with respect to what counts as 'one'. Sutton \& Filip (2016) therefore set a cap on the number of object mass nouns that can occur in a given language, though this cap is straightforwardly compatible with the findings reported here unlike the predictions of Chierchia (2015) and Rothstein (2010).

What can be concluded from these findings is that it is most likely that the amount of object mass nouns in a language is a complex phenomena that has to do with a large number of characteristics of nominal systems rather than just one characteristic as seems to be the case in the analyses of Chierchia (2015) and Rothstein (2010) where number marking and counting constructions respectively are the characteristics of a language's nominal system that most significantly impact the number of object mass nouns. Instead, relative amounts of morphosyntactic environments in which a noun's countability is overtly specified seems to be a more accurate 
predictor.

The set of morphosyntactic environments available must also impact the number of object mass nouns in a language given some environments will be more or less likely to be used in discourse-e.g. a quantifier such as many can be applied to more contexts than a quantifier like dozens of - so the rate at which certain morphosyntactic environments occur will be affected in some why by the semantics of the environments themselves. Future work comparing the types of morphosyntactic environments in which a noun's countability is specified that are available in different languages, along with the relative amounts of object mass nouns, might be able to characterize the impact that particular types of these morphosyntactic environments have on the amount of object mass nouns.

\section{References}

Alexiadou, Artemis. 2011. Plural mass nouns and the morpho-syntax of number. In Mary Byram Washburn, Katherine McKinney-Bock, Erika Varis, Ann Sawyer \& Barbara Tomaszewicz Proceedings of the 28th West Coast Conference on Formal Linguistics. 33-41. Somerville, MA: Cascadilla Press.

Alexiadou, Artemis. 2015. Deriving fake mass nouns. In Thuy Bui \& Deniz Özy1ldız (eds.), Proceedings of the forty-fifth Annual Meeting of the North East Linguistic Society (Volume 45), 11-24. Amherst, MA: GLSA Publications.

Barner, David \& Jesse Snedeker. 2005. Quantity judgments and individuation: Evidence that mass nouns count. Cognition 97(1). 41-66. https://doi.org/10.1016/j.cognition.2004.06.009.

Cheng, Lisa L-S \& Rint Sybesma. 1998. Yi-wan tang, yi-ge tang: Classifiers and massifiers. Tsing-Hua Journal of Chinese Studies 28(3). 385-412.

Chierchia, Gennaro. 1998. Plurality of mass nouns and the notion of "semantic parameter". In Susan Rothstein (ed.), Events and grammar (Studies in Linguistics and Philosophy 7). 53-103. Dordrecht: Kluwer. https://doi.org/10.1007/978-94-011-3969-4.

Chierchia, Gennaro. 2010. Mass nouns, vagueness and semantic variation. Synthesé 174(1). 99149. https://doi.org/10.1007/s11229-009-9686-6.

Chierchia, Gennaro. 2015. How universal is the mass/count distinction? Three grammars of counting. In Audrey Li, Andrew Simpson, and Wei-Tien Dylan Tsai (eds.), Chinese syntax: A crosslinguistic perspective. 147-177. Oxford: Oxford University Press. https://doi.org/10.1093/acprof:oso/9780199945658.003.0006.

Erbach, Kurt. 2020. Object mass nouns. Düsseldorf: Heinrich Heine University dissertation.

Erbach, Kurt, Hana Filip \& Peter R. Sutton. 2019. Bare nouns and the Hungarian mass/count distinction. In Alexandra Silva, Sam Staton, Peter Sutton \& Carla Umbach (eds.), Language, logic, and computation (TbiLLC 2018; Lecture Notes in Computer Science 11456). Dordrecht: Springer.

Erbach, Kurt, Peter R. Sutton, Hana Filip \& Kathrin Byrdeck. 2020. Object mass nouns as arbiter for the mass/count category. Manuscript. Düsseldorf: Heinrich-Heine University.

Gillon, Brendan S. 1999. The lexical semantics of English count and mass nouns. In Evelyne Viegas (ed.), Breadth and depth of semantic lexicons. 19-37. Dordrecht: Springer. https://doi.org/10.1007/978-94-017-0952-1_2.

Gordon, Peter. 1985. Evaluating the semantic categories hypothesis: The case of the count/mass distinction. Cognition 20(3). 209-242. 
Grimm, Scott. 2012. Number and individuation. Palo Alto, CA: Stanford University dissertation.

Grimm, Scott \& Beth Levin. Submitted. Furniture and other artifactual aggregates 1-57. Kouneli, Maria. 2019. Plural marking on mass nouns Evidence from Greek. In Eric Mathieu, Myriam Dali \& Gita Zareikar (eds.), Gender and noun classification. 233-249. Oxford: Oxford University Press.

Krifka, Manfred. 1989. Nominal reference, temporal constitution and quantification in event semantics. In Renate Bartsch, Johan van Benthem \& P. van Emde Boas (eds.), Semantics and contextual expression. 75-115. Dordrecht: Foris.

Krifka, Manfred. 2009. Counting configurations. Proceedings of Sinn und Bedeutung 13. 309-324.

Landman, Fred. 2011. Count nouns - mass nouns, neat nouns - mess nouns. Baltic International Yearbook of Cognition, Logic and Communication 6(1). 1-67.

Landman, Fred. 2016. Iceberg semantics for count nouns and mass nouns: The evidence from portions. The Baltic International Yearbook of Cognition Logic and Communication 11. 1-48.

Li, Xu-Ping. 2011. On the semantics of classifiers in Chinese. Tel Aviv: Bar-Ilan University dissertation.

Link, Godehard. 1983. The logical analysis of plurals and mass terms. In Bäuerle, Rainer, Christoph Schwarze \& Arnim von Stechow (eds.), Meaning, use, and interpretation of language, 127-146. Berlin: De Gruyter.

Muromatsu, Keiko. 2003. Classifiers and the count/mass distinction. In Andrew Simpson \& Audrey-Li Yen-hui (eds.), Functional structure(s), form and interpretation: Perspectives from East Asian languages. 65-96. London: Routledge.

Nemoto, Naoko. 2005. On mass denotations of bare nouns in Japanese and Korean. Linguistics 43(2). 383-413.

Rothstein, Susan. 2010. Counting and the mass/count distinction. Journal of Semantics 27(3). 343397. https://doi.org/10.1093/jos/ffq007.

Rothstein, Susan. 2017. Semantics for counting and measuring. Cambridge, UK: Cambridge University Press.

Schvarcz, Brigitta R. \& Susan Rothstein. 2017. Hungarian classifier constructions, plurality and the mass-count distinction. In Approaches to Hungarian: Volume 15: Papers from the 2015 Leiden Conference. 183-208. Amsterdam: John Benjamins. https://doi.org/10.1075/atoh.15.07sch.

Schwarzschild, Roger. 1996. Pluralities. Dordecht: Springer.

Schwarzschild, Roger. 2011. Stubborn distributivity, multiparticipant nouns and the count/mass distinction. In Suzi Lima, Kevin Mullin \& Brian Smith (eds.) Proceedings of the thirty-ninth Annual Meeting of the North East Linguistic Society (Volume 39). 661-678. Amherst, MA: GLSA Publications.

Soja, Nancy, Susan Carey \& Elizabeth S. Spelke. 1991. Ontological categories guide young children's inductions of word meaning: Object terms and substance terms. Cognition 38(2). 179211.

Sudo, Yasutada. 2015. Countable nouns in Japanese. Manuscript, University College, London.

Sudo, Yasutada. 2016. The semantic role of classifiers in Japanese. Baltic International Yearbook of Cognition, Logic and Communication 11(1). 1-15.

Sutton, Peter R. \& Hana Filip. 2016. Counting in context: Count/mass variation and restrictions on coercion in collective artifact nouns. Semantics and Linguistic Theory (SALT) 26. 350-370. https://doi.org/10.3765/salt.v26i0.3796.

Sutton, Peter R. \& Hana Filip. 2018. Restrictions on subkind coercion in object mass nouns. Pro- 
ceedings of Sinn und Bedeutung 21. 1195-1213.

Tsoulas, George. 2008. On the grammar of number and mass terms in Greek. In Claire Halpert, Jeremy Hartman \& David Hill (eds.), Proceedings of the 2007 Workshop in Greek Syntax and Semantics at MIT (MIT Working Papers in Linguistics 57). 131-46. Cambridge, MA: MIT. 